\section{Sepsis Causing Bacteria and their Mechanisms of Overcoming Host Defense}

\section{Hayk Minasyan*}

Department of Immunology and Microbiology, Private Laboratory, Mamikonyanz, 38-38, Yerevan, Armenia

*Corresponding author: Hayk Minasyan, Department of Immunology and Microbiology, Principal Researcher, Private Laboratory, Mamikonyanz, 38-38, Yerevan, Armenia, Tel: +374 77255295; E-mail: haykminasyan@rambler.ru

Received date: July 13, 2017; Accepted date: July 18, 2017; Published date: July 19, 2017

Copyright: $\odot 2017$ Minasyan $\mathrm{H}$. This is an open-access article distributed under the terms of the Creative Commons Attribution License, which permits unrestricted use, distribution, and reproduction in any medium, provided the original author and source are credited.

Keywords: Sepsis; Pathogens; Phagocytosis; Immunity

\section{Introduction}

Few bacteria cause sepsis in humans. Sepsis causing bacteria have special tools that provide by-passing and overcoming human antibacterial defense. Human innate immunity contains at least six barriers for providing the killing of bacteria and preventing proliferation and dissemination of pathogens (Figure 1).

Infection enters the bloodstream after overcoming tissue barriers (complement, confinement in blood clots, phagocytosis by macrophages, inactivation and killing by NETs and platelets, etc.). In the bloodstream bacteria are killed by oxygen released from erythrocytes. If bacteria survive oxidation, they may enter erythrocytes, proliferate there and form bacterial reservoir inside red blood cells. Massive proliferation of bacteria inside erythrocytes and their release to the bloodstream causes secondary dissemination of pathogens to the tissues and host's immune system tries to prevent that by disseminated intravascular coagulation.

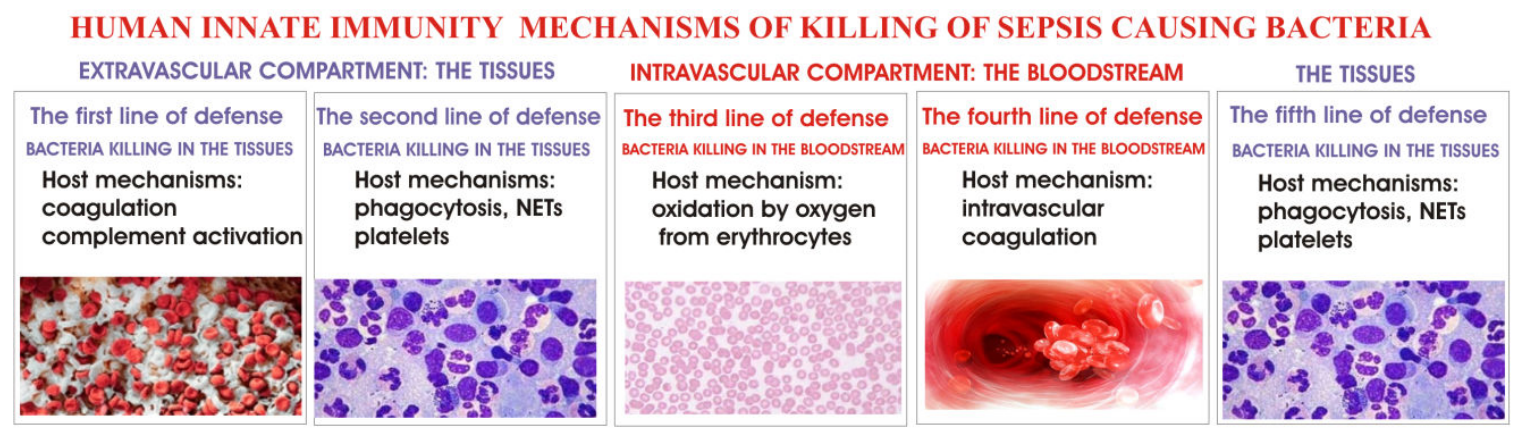

Figure 1: Human innate immunity mechanisms of killing bacteria.

\section{The First Line of Host Defense}

Coagulation in the tissues is the first line of human innate immunity. Blood clot formation not only prevents hemorrhage, but also prevents contamination of the tissues. Integument blood clots are formed by capillary blood and contain enough oxygen for oxidation and killing of bacteria. Blood clots contain relatively few leukocytes, besides, the cells are destroyed by pressure and the clot density makes impossible phagocytosis from the beginning of clot formation. Poured out content of leukocytes may increase bactericidal effect inside blood clots as it happens in case of NETs. In blood clots the concentration of complement proteins are higher than in the blood plasma. Complement proteins may directly kill and opsonize pathogens. Blood clots are a very special trap for bacteria: clot desiccation causes immobilization, squeezing, dehydration of trapped bacteria, besides, bacteria are killed by oxygen and high concentrations of iron. The majority of bacterial species is killed in blood clots and can't overcome this barrier, but sepsis causing bacteria have developed special mechanisms for survival in blood clots (Figure 2). These mechanisms are effective both in blood coagulation in the tissues and disseminated intravascular coagulation in the bloodstream.

\section{The Second Line of Host Defense}

The tissues are the realm of resident macrophages and leukocytes transmigrated from the bloodstream. Bacteria may invade the tissues without causing coagulation. The mechanisms of bacteria killing in the tissues are well-known [1-3]. Bacteria have numerous mechanisms of camouflage, evading recognition, surviving phagocytosis, killing macrophages, proliferating and disseminating in leukocytes, neutralizing NETs, etc. (Figure 2). Sepsis causing bacteria use these mechanisms also in case of secondary invasion of distant tissues by the bloodstream in the final stage of sepsis.

\section{The Third Line of Host Defense}

After overcoming host defense in the tissues, bacteria directly and/or by lymph enter the bloodstream. Phagocytosis in the bloodstream is impossible because leukocytes cannot recognize and engulf bacteria in flowing blood. Bacteria can't proliferate in the bloodstream because of triboelectric charging [4]

Bacteria are recognized and caught in the bloodstream by the phenomenon of electric charge attraction. Erythrocytes are the main 
bactericidal cell in the bloodstream. They catch bacteria by electric charge attraction and kill them by oxygen released from hemoglobin. Electrostatic attraction force keeps bacteria on the surface of erythrocytes; the stimulation and irritation of erythrocyte membrane causes release of oxygen from oxyhemoglobin. Released oxygen is highly reactive and oxidizes and kills bacteria $[4,5]$. The most effective killing of bacteria occurs in the lungs (pulmonary capillaries), bactericidal oxidation is also effective in pulmonary veins, aorta and systemic arteries (where erythrocytes contain enough oxyhemoglobin). Bacteremia in arterial and venous blood has different consequences. Bacteria easily survive in the venous blood because of the lack of oxygen in venous blood erythrocytes, whereas few bacteria survive oxidation in the lungs, pulmonary veins and systemic arteries. For development of sepsis bacteria should survive oxidation in the lungs and arterial blood [6].

Sepsis causing bacteria have effective anti-oxidative mechanisms for neutralizing and surviving intensive oxidation (Figure 2). The main mechanisms are facultative anaerobe respiration, capsule expression, production of anti-oxidative enzymes (catalase, superoxide dismutase, glutathione, etc.). If bacteria survive oxidation on the surface of erythrocytes, they may produce pore-forming factors, penetrate through erythrocyte membranes and proliferate inside erythrocytes. Bacterial reservoir inside erythrocytes is unreachable for immune complexes, antibiotics and other bactericidal substances. If bacteria provoke massive release of oxygen from oxyhemoglobin, the consequences are dramatic: released oxygen impairs humoral regulation and homeostasis by oxidation of blood plasma components (proteins, hormones, peptides, amino acids, vitamins, other biologically active substances), besides, premature release of oxygen causes tissue hypoxia and provokes disseminated intravascular coagulation with tissue ischemia and necrosis. The release of oxygen from erythrocytes to arterial blood is one of important co-factors of septic shock and multiple organ dysfunctions [7].

\section{HUMAN INNATE IMMUNITY AND THE SURVIVAL MECHANISMS OF SEPSIS CAUSING BACTERIA}

Host's first and fourth lines of defense
Kinases
Fibrinogen-degrading proteinases
Cysteine proteinases
Subtilases
Plasminogen sequestration
Superantigen-like protein 10 (SSL10)
Complement inhibitor factor H (FH)

Complement inhibitor factor $\mathrm{H}$ (FH) Capsule expression

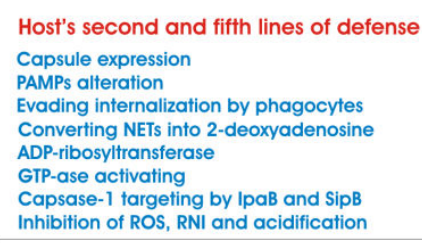

Host's second and fifth lines of defense Capsule expression PAMPs alteration Evading internalization by phagocytes Converting NETs into 2-deoxyadenosine ADP-ribosyltransferase GTP-ase activating Capsase-1 targeting by IpaB and SipB Inhibition of ROS, RNI and acidification

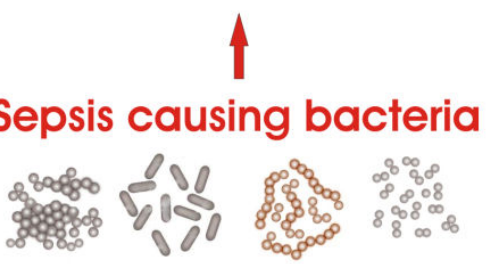

Sepsis causing bacteria

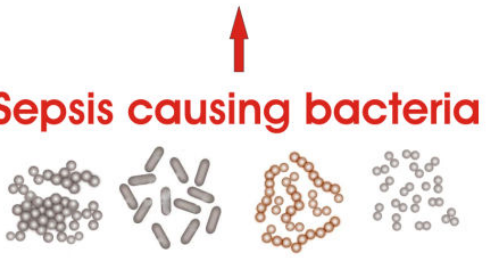

Host's third line of defense Capsule expression Facultative anaerobe respiration Catalase production Superoxide dismutase production Glutathione production

Decreasing metabolic activity and growth Production of pore-forming enzymes

\section{I}

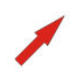

Figure 2: Human innate immunity and survival mechanisms of sepsis causing bacteria.

\section{The Fourth and Fifth Lines of Host Defense}

The last two lines of innate immunity defense (disseminated intravascular coagulation and bacteria killing in the tissues) are the least effective host defense and cannot stop proliferation and dissemination of infection.

The bacteria, that have survived coagulation during primary invasion of host tissues, successfully escape blood clots in this final stage of host defense. Moreover, disseminated intravascular coagulation causes serious circulatory problems that may be fatal for hosts.

For entering the bloodstream, bacteria should overcome tissue bactericidal mechanisms (phagocytosis, NETs, etc.). Sepsis causing bacteria that enter the bloodstream after overcoming tissue bactericidal mechanisms, are resistant to phagocytosis, NETs and direct bactericidal action of platelets in distant tissues contaminated by blood flow [7].

\section{Conclusion}

Bactericidal mechanisms of human innate immunity are effective and provide protection against the majority of bacterial pathogens. A very limited number of bacteria have developed tools for by-passing and overcoming human innate immunity. Sepsis-causing bacteria are among the pathogens that can overcome human innate immunity. Most episodes of bacteremia spontaneously resolve because pathogens are killed by oxidation on the surface of erythrocytes. Bacterial resistance to oxidation is indispensable for survival of pathogens in the bloodstream. Bacterial proliferation inside erythrocytes and provoked by bacteria abundant premature release of oxygen from erythrocytes to arterial blood are critical events for development of sepsis and septic shock. Bacteria inside erythrocytes are unreachable for immune complexes, antibiotics and other bactericidal substances; the oxidation of plasma components causes multiple organ dysfunctions by destroying humoral regulation and homeostasis and provoking disseminated intravascular coagulation with tissue ischemia, hypoxia and necrosis. 
Citation: Minasyan H (2017) Sepsis Causing Bacteria and their Mechanisms of Overcoming Host Defense. J Infect Dis Diagn 2: 113.

Page 3 of 3

\section{References}

1. Aderem A (2003) Phagocytosis and the inflammatory response. J Infect Dis $187: 340-345$.

2. Hoffmann J, Akira S (2013) Innate immunity. Curr Opin Immunol 25: $1-3$

3. Travis J (2009) Origins. On the origin of the immune system. Science 324 580-582.

4. Minasyan H (2014) Erythrocyte and blood antibacterial defense. Eur Microbiol Immunol 4: 138-143.
5. Minasyan H (2014) Erythrocyte and leukocyte: two partners in bacteria killing. Int Rev Immunol 33: 490-497.

6. Minasyan H (2016) Mechanisms and pathways for the clearance of bacteria from blood circulation in health and disease. Pathophysiology 23: 61-66.

7. Minasyan H (2017) Sepsis and septic shock: Pathogenesis and treatment perspectives. J Crit Care 40: 229-242. 